\title{
Targeting Policy for Obesity Prevention: Identifying the Critical Age for Weight Gain in Women
}

\author{
Trevor J. B. Dummer, ${ }^{1}$ Sara F. L. Kirk, ${ }^{2}$ Tarra L. Penney, ${ }^{2}$ Linda Dodds, ${ }^{3}$ and Louise Parker ${ }^{1}$ \\ ${ }^{1}$ Population Cancer Research Program, Department of Pediatrics, Dalhousie University, 1494 Carlton Street, P.O. Box 15000, \\ Halifax, NS, Canada B3H 4R2 \\ ${ }^{2}$ Applied Research Collaborations for Health, School of Health and Human Performance, Dalhousie University, \\ 1318 Robie Street, Halifax, NS, Canada B3H 3E2 \\ ${ }^{3}$ Perinatal Epidemiology Research Unit, Departments of Obstetrics and Gynaecology and Pediatrics, Dalhousie University, \\ IWK Health Centre, 5850/5980 University Avenue, P.O. Box 9700, Halifax, NS, Canada B3K 6R8
}

Correspondence should be addressed to Trevor J. B. Dummer, trevor.dummer@dal.ca

Received 22 August 2011; Revised 27 October 2011; Accepted 27 October 2011

Academic Editor: Ruth H. Striegel-Moore

Copyright (C) 2012 Trevor J. B. Dummer et al. This is an open access article distributed under the Creative Commons Attribution License, which permits unrestricted use, distribution, and reproduction in any medium, provided the original work is properly cited.

\begin{abstract}
The obesity epidemic requires the development of prevention policy targeting individuals most likely to benefit. We used selfreported prepregnancy body weight of all women giving birth in Nova Scotia between 1988 and 2006 to define obesity and evaluated socioeconomic, demographic, and temporal trends in obesity using linear regression. There were 172,373 deliveries in this cohort of 110,743 women. Maternal body weight increased significantly by $0.5 \mathrm{~kg}$ per year from 1988 , and lower income and rural residence were both associated significantly with increasing obesity. We estimated an additional 82,000 overweight or obese women in Nova Scotia in 2010, compared to the number that would be expected from obesity rates of just two decades ago. The critical age for weight gain was identified as being between 20 and 24 years. This age group is an important transition age between adolescence and adulthood when individuals first begin to accept responsibility for food planning, purchasing, and preparation. Policy and public health interventions must target those most at risk, namely, younger women and the socially deprived, whilst tackling the marketing of low-cost energy-dense foods at the expense of healthier options.
\end{abstract}

\section{Introduction}

The World Health Organisation (WHO) estimates that globally 1.5 billon adults are overweight and 500 million are obese [1]. Almost 25\% of Canadians are currently obese, with an estimated 1 in 10 premature adult deaths attributable directly to obesity [2]. Obesity rates in Nova Scotia have more than doubled since $1985[3,4]$. With 20\% of Nova Scotians classed as obese and a further $35 \%$ classed as overweight, the Provincial rate is substantially higher than the Canadian average of $16 \%$ and $32 \%$ for obese and overweight, respectively [5]. Further, overweight and obesity rates are higher in Nova Scotian women than men [3]. These trends have important implications for population health and health system planning.

Obesity is a serious public health issue. It is a risk factor for premature mortality from a number of causes, such as hypertension, coronary artery disease, and type 2 diabetes, as well as many types of cancer $[2,6]$.

While the causes of the obesity epidemic are simple in principle-an imbalance between energy consumption (diet) and energy expenditure (physical activity) —in reality the problem is complex and the result of an interaction between genetic, lifestyle, and environmental factors [7]. A greater understanding of policy options and of potential target populations for obesity prevention is therefore necessary.

Understanding the parameters of maternal weight status is of critical importance for a number of reasons. Maternal obesity is related to a significantly higher risk for complications during pregnancy, including a higher rate of delivery and surgical difficulties, hypertension, thromboembolism, and gestational diabetes which also contribute to fetal complications such as congenital malformations, macrosomia, and antepartum stillbirth $[8,9]$. Furthermore, pregnancy 
often alters the weight gain trajectory of an individual by increasing the likelihood of postpartum weight retention if gestational weight gain is greater than the recommended range $[10,11]$. This cycle may then be repeated with each additional pregnancy as increases in maternal body weight can occur with parity, further perpetuating the obesity epidemic [12]. Obese women are also less likely to breast-feed for mechanical as well as physiological reasons, removing a fundamental safeguard against long-term weight gain for themselves and their children [13]. These early life factors, among many others, make it more likely that the children of obese mothers will themselves be overweight or obese, or at greater risk of becoming overweight or obese adolescents and adults [14]. In particular, the relatively new concept of assortative weight gain suggests an important role of the mother in influencing body mass index (BMI) of daughters, with a weaker relationship seen between fathers and sons [14]. Studies have identified that women of childbearing age are at a particularly high risk of weight gain and future obesity [15]. Amongst this group, the highest risk has been identified in the $25-45$ age range [16].

Identifying the target for policy approaches requires some consideration. For example, prevention of obesity in children is an important element of obesity prevention [17], but targeting children without also addressing parental obesity may not be an effective or viable solution. Therefore, targeting obesity prevention policies at childbearing women could offer a dual benefit - to both the mother and the child. However, information on the parameters of maternal weight status is limited, particularly in terms of identifying critical periods for weight gain.

The purpose of this study was therefore to identify the socioeconomic, demographic, and temporal trends of maternal prepregnancy bodyweight for Nova Scotian women. Specifically we analyzed changes in prepregnancy body weight of women giving birth in Nova Scotia between 1988 and 2006 to identity the critical window for excessive weight gain in this substantial cohort of women. Our overall aim was to understand how weight gain varies at different age points in order to understand better the role of lifecourse factors that influence obesity risk.

\section{Materials and Methods}

2.1. Data. Canada has no national surveillance system in place to assist in the identification of overweight or obese women giving birth. Therefore the development of evidencebased public health policy is problematic. The Canadian Perinatal Surveillance System (CPSS) [18] is the closest system and collects 27 health outcomes however height, weight, or waist circumference of the mother is not included. In Nova Scotia, the Atlee Perinatal Database (NSAPD) records information on pregnancy outcomes and was used to analyse trends in maternal prepregnancy weight. Ethical approval was obtained from the Research Ethics Board at the IWK Health Centre, Halifax, Nova Scotia.

The total female population of Nova Scotia in 2010 was approximately 485,000. The NSAPD represents a sizeable fraction of this Nova Scotian female population, with prepregnancy self-reported body weight from over 110,000 women, and a total of 196,209 individual deliveries, collected between January 1st 1988 and December 31st 2006. Of these deliveries 23,829 (12\%) were excluded from this analysis, mostly because prepregnancy weight was not recorded but a very small number had no parity information recorded, resulting in a total analysis dataset of 110,743 women with 172,373 deliveries and prepregnancy body weight measurements. No other covariates beyond age and parity that are relevant to obesity risk were available in the NSAPD, although we were able to use the residential postal code for each woman to define urban/rural residence and assign an indicator of socioeconomic status from census information.

2.2. Outcome Measures. Self-reported prepregnancy body weight in kilograms $(\mathrm{kg})$, taken from the standard provincial prenatal form used in Nova Scotia, was used as the principal outcome measure. For all women this information was collected at their first physician visit where their pregnancy was confirmed. This was used as a continuous and discrete variable; for the discrete comparisons, individuals were classified as underweight $(<55 \mathrm{~kg})$, normal weight $(55-75 \mathrm{~kg})$, overweight $(>75-90 \mathrm{~kg})$, or obese $(>90 \mathrm{~kg})$ using the same classifications developed by Robinson et al. in an analysis of body weight changes in the NSAPD [8]. Demographic variables analysed were age, by year and in six categories $(<20,20-24,25-29,30-24,35-39, \geq 40)$, parity, income decile (derived from census data), and location (urban versus rural residence).

2.3. Analysis. Descriptive statistics were used to explore temporal, socioeconomic, and demographic trends in body weight. Multivariate linear regression modelling of body weight was conducted for the following predictor variables: year of birth (of the offspring), mother's age (continuous, six categories), socioeconomic status (income deciles), and location (urban/rural). These data relate to prepregnancy body weight at specific pregnancies in a longitudinal cohort of women, and therefore the analysis was clustered on maternal identifier, and robust estimates of variance were used [19]. The overall significance of each regression model was assessed using the $F$-test; significance of individual coefficients was assessed through inspection of the $t$-statistic and the $95 \%$ confidence intervals (CIs) for the coefficients. Statistical analysis was performed using STATA 10 [20].

\section{Results}

Prepregnancy body weight was recorded for 172,373 deliveries to women resident in Nova Scotia between 1988-2006. There were very few deliveries $(<0.1 \%)$ to women aged over 45 years in the cohort. Overall, the average age at first birth was 26 years, increasing from 25 years in 1988 to 27 years in 2006, highlighting the trend for women having babies later in life. Figure 1 shows the change in median body weight over time for primiparous and multiparous women, which represents a steep upward trend over the last two decades. Multiparous women were consistently heavier than primiparous women, and the difference in median body 
TABLE 1: Linear regression modelling of body weight in relation to demographic factors (i) unadjusted, (ii) adjusted for parity, and (iii) adjusted for parity and all other variables.

\begin{tabular}{|c|c|c|c|c|c|c|c|c|c|c|c|}
\hline \multirow{2}{*}{ Variable } & \multicolumn{2}{|c|}{ Body weight (kg) } & \multicolumn{3}{|c|}{ Unadjusted } & \multicolumn{3}{|c|}{ Adjusted (parity) } & \multicolumn{3}{|c|}{ Adjusted (all variables) } \\
\hline & Mean & Median & Coefficient & $95 \% \mathrm{CI}$ & $P$ & Coefficient & $95 \% \mathrm{CI}$ & $P$ & Coefficient & $95 \% \mathrm{CI}$ & $P$ \\
\hline Year & - & - & 0.53 & $0.51-0.55$ & $<0.01$ & 0.53 & $0.52-0.55$ & $<0.01$ & 0.53 & $0.51-0.54$ & $<0.01$ \\
\hline Age group & & & & & $<0.01$ & & & $<0.01$ & & & $<0.01$ \\
\hline$<20$ & 59.4 & 56.7 & - & - & & - & - & & - & - & \\
\hline $20-24$ & 65.2 & 61.2 & 5.84 & $5.57-6.12$ & & 5.70 & $5.42-5.98$ & & 5.42 & $5.13-5.70$ & \\
\hline $25-29$ & 67.1 & 63.5 & 7.74 & $7.47-8.02$ & & 7.52 & $7.23-7.81$ & & 7.46 & $7.16-7.75$ & \\
\hline $30-34$ & 67.2 & 63.5 & 7.86 & $7.57-8.14$ & & 7.57 & $7.26-7.89$ & & 7.27 & $6.94-7.59$ & \\
\hline $35-39$ & 67.6 & 63.5 & 8.23 & $7.89-8.57$ & & 7.90 & $7.52-8.27$ & & 7.19 & $6.80-7.58$ & \\
\hline$\geq 40$ & 68.0 & 64.4 & 8.62 & 7.99-9.25 & & 8.24 & $7.58-8.90$ & & 7.06 & $6.39-7.72$ & \\
\hline Income (SES) & - & - & -0.17 & $-0.20--0.14$ & $<0.01$ & -0.17 & $-0.21--0.14$ & $<0.01$ & -0.27 & $-0.30--0.24$ & $<0.01$ \\
\hline Urbanicity & & & & & $<0.01$ & & & $<0.01$ & & & $<0.01$ \\
\hline Urban & 66.0 & 62.6 & - & - & & - & - & & - & - & \\
\hline Rural & 66.9 & 63.5 & 0.82 & $0.63-1.01$ & & 0.75 & $0.56-0.94$ & & 1.49 & $1.30-1.67$ & \\
\hline
\end{tabular}

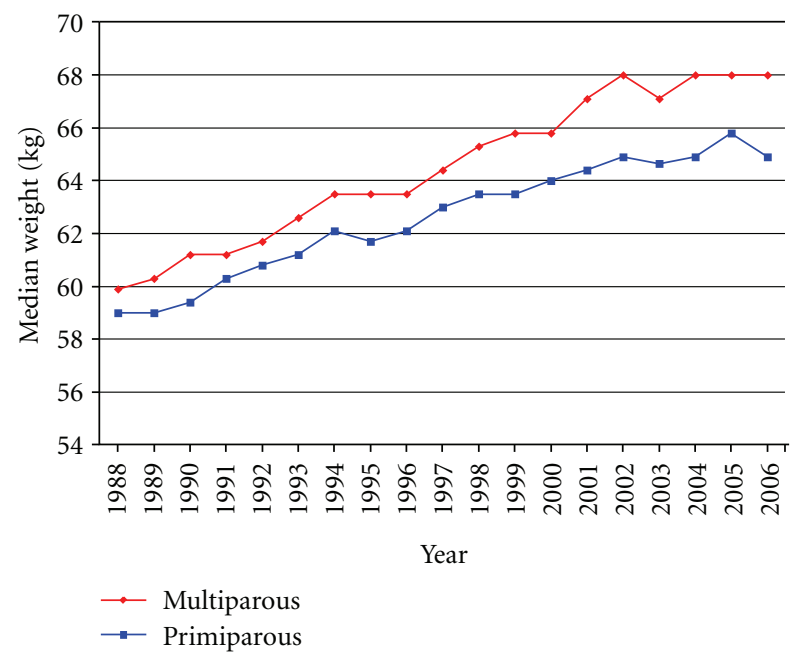

Figure 1: Median prepregnancy bodyweight, primiparous, and multiparous women, 1988-2006.

weight between these groups showed evidence of widening in the latter years of the study. The percentage of individuals in each weight category by four-year time periods displays a clear trend, with notable increases in the number of overweight/obese women and a fall in the percentage of those women classed as underweight (Figure 2). During the first time period (1988-1991) the overweight and obesity prevalence was $14 \%$, whereas by the final time period (20042006), the prevalence had increased to $31 \%$. Extrapolating these percentages to the total adult female population of Nova Scotia in 2010 (485,000 women) the province had an estimated 150,000 overweight or obese women, compared to just 68,000 women overweight or obese if the 1988-1991 obesity rate still applied.

Table 1 shows the results from the linear regression modeling of body weight socioeconomic and demographic risk factors (adjusted and unadjusted). For continuous variables

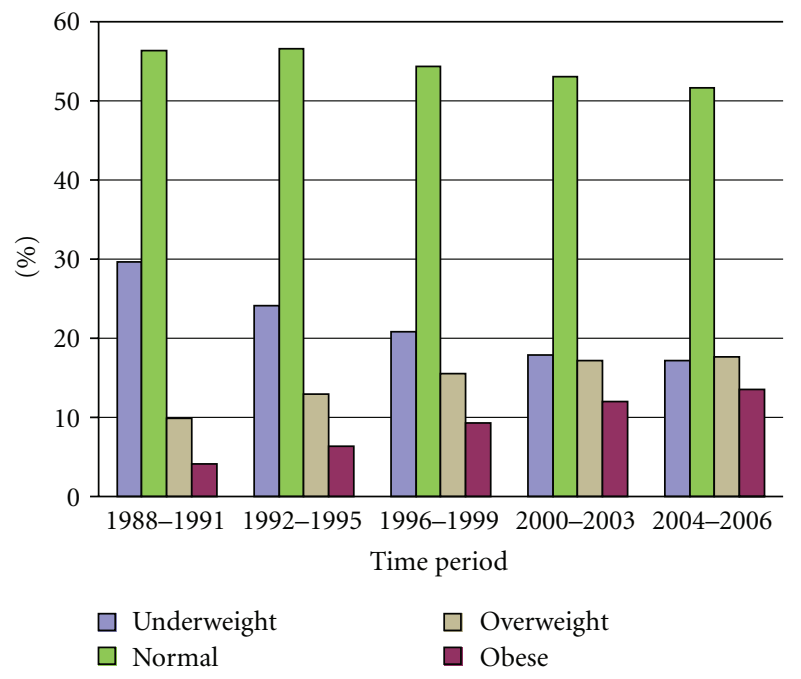

Figure 2: Percentage of women classified as underweight $(<55 \mathrm{~kg})$, normal weight $(55-75 \mathrm{~kg})$, overweight $(>75-90 \mathrm{~kg})$, and obese (>90 kg) by time period, 1988-2006.

(year and income deciles), the coefficient represents the weight change (in $\mathrm{kg}$ ) for every unit change in either year or income decile. For categorical variables the coefficient represents the weight change in $\mathrm{kg}$ for each category, compared to the mean weight in the baseline category (i.e., the coefficient for the constant). Body weight increased by $0.5 \mathrm{~kg}$ each year on average across the duration of the study period. In each age category women were between $6 \mathrm{~kg}$ and $8 \mathrm{~kg}$ heavier than the youngest age group of women aged $<20$ years, even after adjusting for parity. Although women aged $>40$ years of age were heavier than women in the younger age groups, the adjusted regression model (Table 1) suggested that weight differences in women in age categories older than 29 years were not markedly different and the largest increase in weight was in the 20-24 years group. Body weight declined by $0.17 \mathrm{~kg}$ for each increase in income decile, compared to 


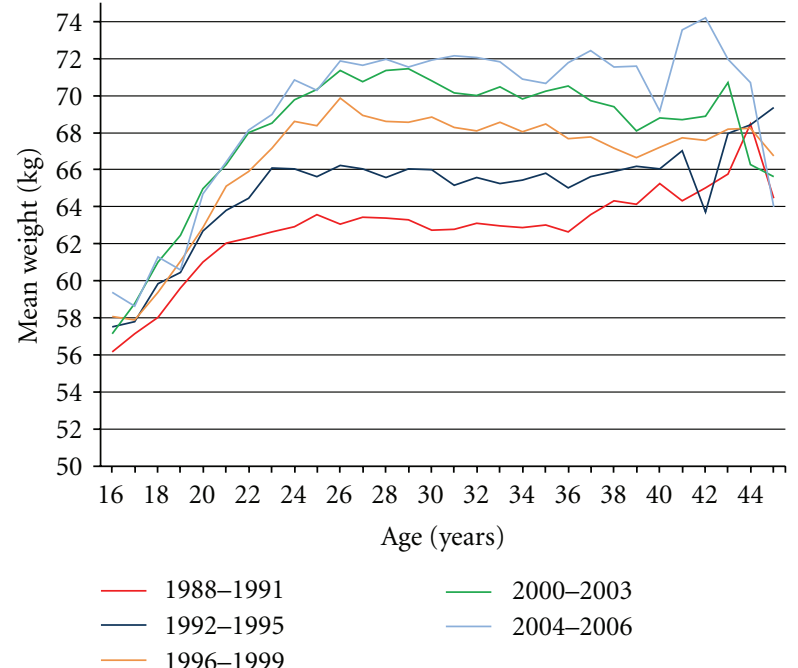

FIGURE 3: Change in maternal prepregnancy bodyweight by year of age and time period, age 16 to 45 years.

the baseline category representing the lowest income group. Women living in rural areas were also significantly heavier than women in urban areas, especially after adjustment for other variables in the model.

Figure 3 shows the change in mean maternal prepregnancy weight by year of age for ages 16 to 45 years by time period. For all time periods average weight increased steadily between ages 16 to 24 but from age 24 upwards weight increased more slowly until women were in their forties. The increase in mean body weight between ages 16 and 24 years was sharper in the later time periods compared to 1988-2001. The pattern in change in weight by year of age was similar for primiparous and multiparous women. Linear regression modelling of weight difference by year of age is presented in Table 2. The modelled weight difference is the weight change with respect to the weight at the baseline age 16 years. There was an increase of between $1 \mathrm{~kg}$ and $1.5 \mathrm{~kg}$ in weight with respect to the weight in each previous year of age until age 19. The largest increase in weight difference between each year of age $(2.2 \mathrm{~kg})$ was between ages 19 and 20 years. There was very little variation in weight difference after age 24 years of age, except for the much older ages, but the sample in these older ages was small, and the $95 \%$ CIs indicated generally that these findings were insignificant.

\section{Discussion}

The purpose of this study was to evaluate the socioeconomic, demographic, and temporal trends in obesity in women residing in the Canadian province of Nova Scotia to identify the critical period for weight gain. During the course of this study, body weight in this large sample of the female population increased significantly, and results showed similar socioeconomic and demographic correlates to obesity as seen from other studies [21-23]. Lower socioeconomic status and living in rural areas were associated significantly with higher body weights. The overall upward temporal trend for weight in Nova Scotian women echoes the increases in overweight and obesity rates over the past few decades worldwide $[1,24]$. Although prepregnancy weight increased with age even after adjusting for parity, the highest weight increases were primarily in the younger women aged below 24 years of age. The largest single increase in weight by year of age was between 19 and 20 years $(2.2 \mathrm{~kg})$, and there was very little difference in mean prepregnancy weight in women aged between 26 and 40 years old. Although the heaviest women were generally aged over 40 years of age, the number of women in this age group was relatively small, as reflected in the wide confidence intervals in the regression modelling. Therefore, weight gain was highest for the younger women in the "transition age group" from adolescence into adulthood: the age when they will be taking responsibility for planning, purchasing, and preparing meals for the first time.

Over the course of this study overweight and obesity prevalence more than doubled from $14 \%$ in $1988-1991$ to $31 \%$ in 2004-2006. These changes suggest that, in 2010, an estimated 150,000 women in Nova Scotia were overweight or obese, representing an additional 82,000 overweight/obese women compared to the number expected from observed rates of obesity two decades ago. Given the clear association of many health risks with obesity [24], this substantial increase in the overweight and obese population will have major implications for health service provision and utilization and healthy public policy [25].

The study data provide a much needed evidence base with which to support public health policy development and targeted public health interventions, information that is currently lacking in Canada due to the limited availability of surveillance data regarding maternal weight status. Our analysis suggests that the critical age for weight gain in this large cohort of women was in the younger age groups, and there was little change in mean weight of women beyond age 24 years. These trends are of importance to public health planning and policy since maternal prepregnancy overweight and excessive gestational weight gain are recognized as contributing factors to child obesity [26-28]. These findings have implications for the intergenerational transmission of weight gain and highlights the unique role that women of childbearing age may have as both a contributor to and a potential solution for the obesity epidemic [14]. Excessive weight gain before and during pregnancy, particularly when subsequently retained postpartum, influences the health of both mother and baby [10-12]. Excessive weight gained during an individual's early twenties carries with it a range of health risk factors, including cancer and other chronic diseases, that they will carry into middle and older age. Prevention efforts targeted at women preconceptually, particularly young adult women, may therefore be necessary to reverse these trends. However, this is not a group typically targeted for interventions to prevent weight gain.

The transition age between adolescence and adulthood is a time of increasing obesity, especially for specific racial/ ethnic populations [29]. Our findings suggest that this age is critically important for obesity in women. The identification of this at risk age group provides an important focus for policy development and prevention intervention design. 
TABLE 2: Change in maternal prepregnancy bodyweight $(\mathrm{kg})$ compared to the reference weight by year of age, unadjusted and adjusted for parity and year of child's birth.

\begin{tabular}{|c|c|c|c|c|c|c|}
\hline \multirow{2}{*}{ Age } & \multicolumn{2}{|r|}{ Weight $(\mathrm{kg})$} & \multicolumn{2}{|c|}{ Unadjusted } & \multicolumn{2}{|c|}{ Adjusted } \\
\hline & Mean & Change by year of age & Weight difference ${ }^{1}(\mathrm{~kg})$ & $95 \% \mathrm{CI}$ & Weight difference ${ }^{1}(\mathrm{~kg})$ & $95 \%$ CI \\
\hline 16 & 57.3 & & - & - & - & - \\
\hline 17 & 57.8 & 0.5 & 0.5 & $-0.3-1.4$ & 0.4 & $-0.5-1.2$ \\
\hline 18 & 59.4 & 1.6 & 2.2 & $1.3-3.0$ & 1.9 & $1.1-2.7$ \\
\hline 19 & 60.6 & 1.2 & 3.3 & $2.5-4.1$ & 2.9 & $2.1-3.7$ \\
\hline 20 & 62.8 & 2.2 & 5.5 & $4.7-6.3$ & 4.9 & $4.1-5.7$ \\
\hline 21 & 64.3 & 1.5 & 7.0 & $6.2-7.8$ & 6.2 & $5.4-7.0$ \\
\hline 22 & 65.1 & 0.8 & 7.9 & $7.1-8.7$ & 7.0 & $6.2-7.9$ \\
\hline 23 & 66.0 & 0.9 & 8.7 & $7.9-9.5$ & 7.9 & $7.1-8.7$ \\
\hline 24 & 66.6 & 0.6 & 9.3 & $8.5-10.1$ & 8.7 & $7.9-9.5$ \\
\hline 25 & 66.7 & 0.1 & 9.4 & $8.6-10.2$ & 8.7 & $7.9-9.5$ \\
\hline 26 & 67.2 & 0.5 & 10.0 & $9.2-10.7$ & 9.3 & $8.5-10.1$ \\
\hline 27 & 67.1 & -0.1 & 9.8 & $9.0-10.6$ & 9.0 & $8.2-9.8$ \\
\hline 28 & 67.0 & -0.1 & 9.8 & $9.0-10.5$ & 9.0 & $8.2-9.7$ \\
\hline 29 & 67.3 & 0.3 & 10.0 & $9.2-10.8$ & 9.0 & $8.2-9.8$ \\
\hline 30 & 67.2 & -0.1 & 9.9 & $9.1-10.7$ & 8.8 & $8.0-9.6$ \\
\hline 31 & 67.0 & -0.2 & 9.7 & $9.0-10.5$ & 8.4 & $7.6-9.2$ \\
\hline 32 & 67.2 & 0.2 & 9.9 & $9.1-10.7$ & 8.5 & $7.7-9.3$ \\
\hline 33 & 67.4 & 0.2 & 10.2 & $9.4-10.9$ & 8.6 & $7.8-9.4$ \\
\hline 34 & 67.2 & -0.2 & 9.9 & $9.1-10.7$ & 8.2 & $7.3-9.0$ \\
\hline 35 & 67.6 & 0.4 & 10.3 & $9.5-11.1$ & 8.4 & $7.6-9.3$ \\
\hline 36 & 67.4 & -0.2 & 10.1 & $9.3-11.0$ & 8.2 & $7.4-9.1$ \\
\hline 37 & 67.8 & 0.4 & 10.5 & $9.6-11.4$ & 8.5 & $7.6-9.4$ \\
\hline 38 & 67.6 & -0.2 & 10.4 & $9.4-11.3$ & 8.3 & $7.3-9.2$ \\
\hline 39 & 67.4 & -0.2 & 10.1 & $9.1-11.1$ & 7.8 & $6.8-8.8$ \\
\hline 40 & 67.4 & 0 & 10.1 & $9.0-11.2$ & 7.8 & $6.7-8.9$ \\
\hline 41 & 68.5 & 1.1 & 11.2 & $9.9-12.5$ & 8.7 & $7.4-10.1$ \\
\hline 42 & 68.2 & -0.3 & 10.9 & $9.3-12.6$ & 8.5 & $6.9-10.1$ \\
\hline 43 & 69.2 & 1 & 11.9 & $9.6-14.2$ & 9.3 & $7.0-11.6$ \\
\hline 44 & 68.3 & -0.9 & 11.0 & $8.3-13.7$ & 8.1 & $5.4-10.9$ \\
\hline 45 & 66.2 & -2.1 & 8.9 & $5.8-12.1$ & 6.5 & $3.3-9.8$ \\
\hline 46 & 65.4 & -0.8 & 8.1 & $1.7-14.6$ & 5.8 & $-0.6-12.2$ \\
\hline 47 & 65.0 & -0.4 & 7.7 & $0.2-15.3$ & 4.5 & $-3.0-12.0$ \\
\hline 48 & 71.4 & 6.4 & 14.1 & $-3.1-31.4$ & 9.9 & $-10.6-30.5$ \\
\hline 49 & 62.7 & -8.7 & 5.4 & $-1.9-12.8$ & 3.3 & $-5.0-11.6$ \\
\hline 50 & 77.5 & 14.8 & 20.2 & $4.3-36.1$ & 17.7 & $2.5-32.9$ \\
\hline
\end{tabular}

${ }^{1}$ Weight difference compared to baseline weight at age 16 years.

Women at this transition age are generally characterised as newly independent and gaining responsibility for family shopping, food choices, and meal planning, but are typically not an age group targeted for intervention. At this age incomes are generally lower, and issues around food insecurity are likely to be heightened. Social and economic resources and food costs influence individual food choices, and energy-dense foods are typically the lowest cost option [30]. These foods contribute significantly to the obesity epidemic [30]. Many young adults, by virtue of socioeconomic circumstances, are therefore vulnerable to the marketing of low-cost energy-dense and obesogenic foods. We suggest that policy to tackle the obesity epidemic is focused both at those most at risk-young women at the transition age-whilst also tackling the food supply industry. The food industry continues to promote and market the availability of low-cost energy-dense foods, at the expense of healthier options, which are generally more expensive [30]. By contrast, more costly but healthier options are much less appealing or practical to individuals living under economic constraints, experiencing food insecurity, and who may not have much experience in food preparation. Targeted education and supportive public policies may encourage individuals to perceive these types of products as more desirable, but the food industry needs to ensure that these foods are also affordable and readily available. 
Weight reduction for obese and overweight people is inherently problematic, and such interventions are rarely successful within the limitations of our current health system [15]. Thus, it is crucial that public health strategies focus on prevention of excessive weight gain. These should promote an integrated approach to food, leisure, transport, and built environment policy and planning, developed in conjunction with public health strategies targeting at-risk individuals and at-risk communities. This approach requires the identification of critical at-risk age groups for weight gain, as identified in this study, in order to provide a focus for prevention interventions and targeted policy initiatives. Our findings of increased obesity in rural areas and the more socially disadvantaged communities suggest that these should also be the focus for targeted interventions and prevention activities.

The use of self-reported body weight in this analysis is a limitation, although given that body weight is often underreported, this is likely to lead to an underestimation of the problem, making these findings all the more concerning. Further the NSAPD does not record height, and therefore it was not possible to calculate BMI, which is the usual indicator of obesity. However, maternal height distribution is unlikely to vary substantially between communities across Nova Scotia; therefore the use of the distribution of body weight as a surrogate for the distribution of obesity is reasonable and has been used elsewhere [8]. This study analysed change in body weight distribution over time (1988-2006), and in the absence of any substantial shifts in population height over short periods of time, there would be no advantage to using BMI since change in body size over time in the absence of changes in height is measured equally well by change in body weight.

These results relate to a subset of the population, women of childbearing age giving birth, derived from the NSAPD. However, this sample provides body weight data related to 172,373 deliveries (over 100,000 women) and is geographically representative of the whole female population. Therefore, the trends described in this study relate to a representative proportion of the Nova Scotia population, and, further, there is no reason to suspect that the body weight increases in this subset of the population have not been similarly experienced by other groups of women. In the absence of any alternative dataset of a comparable size and level of detail that is representative of the provincial female population, these data provide very valuable information for policy making and prevention initiatives.

\section{Conclusions}

Our findings add to the literature by clearly demonstrating the inexorable rise in body weight that has occurred in the last two decades in a subgroup of the population of childbearing age that precedes pregnancy. These findings support the targeting of prevention initiatives at younger age groups, as well as those from socially disadvantaged and rural communities. Younger women are at a transition age, newly independent, gaining responsibility for food choices and experiencing food insecurity, and therefore extremely vulnerable to the marketing efforts of a food industry which promotes the consumption of low-cost energy-dense foods over and above healthier but more costly options. However, this age group is not a traditional focus for health promotion and we suggest that policy options need to go beyond promoting healthy eating for individuals to tackling the food supply industry and its overemphasis on obesogenic foods. Our findings offer insight of relevance to public health planners and policy makers and suggest that obesity prevention initiatives aimed at women of childbearing age, both before and afterconception, may hold promise to deal with the consequences of the obesity epidemic, whilst also supporting strategies to deal with the causes.

\section{Conflict of Interests}

The authors declare that there is no conflict of interests.

\section{Acknowledgments}

The authors acknowledge the following funding: S. F. L. Kirk and T. L. Penney were supported by funds from the IWK Health Centre, Halifax (Scholar Award to S. F. L. Kirk). L. Parker acknowledges support from the IWK Health Centre, Halifax (Establishment Grant).

\section{References}

[1] World Health Organisation. WHO, Obesity and overweight (Factsheet), 2011, http://www.who.int/mediacentre/factsheets/ fs311/en/.

[2] D. C. W. Lau, J. D. Douketis, K. M. Morrison, I. M. Hramiak, A. M. Sharma, and E. Ur, "2006 Canadian clinical practice guidelines on the management and prevention of obesity in adults and children [summary]," Canadian Medical Association Journal, vol. 176, no. 8, pp. S1-13, 2007.

[3] M. Tjepkema, "Adult obesity," Health Reports, vol. 17, no. 3, pp. 9-25, 2006.

[4] R. Colman, "Cost of obesity in Nova Scotia," 2000, http:// www.gpiatlantic.org/pdf/health/obesity/ns-obesity.pdf.

[5] Statistics Canada, "Self-reported overweight and obesity, adults aged 18 or older, by selected characteristics, Canada excluding the territories," The Daily, Statistics Canada, 2008, http://www.statcan.gc.ca/daily-quotidien/080618/t080618aeng.htm.

[6] S. Y. Pan, K. C. Johnson, A.-M. Ugnat, S. W. Wen, and Y. Mao, "Association of obesity and cancer risk in Canada," American Journal of Epidemiology, vol. 159, no. 3, pp. 259-268, 2004.

[7] K. M. Booth, M. M. Pinkston, and W. S. C. Poston, "Obesity and the built environment," Journal of the American Dietetic Association, vol. 105, no. 5, supplement 1, pp. S110-S117, 2005.

[8] H. E. Robinson, C. M. O’Connell, K. S. Joseph, and N. L. McLeod, "Maternal outcomes in pregnancies complicated by obesity," Obstetrics \& Gynecology, vol. 106, no. 6, pp. 13571364, 2005.

[9] C. K. H. Yu, T. G. Teoh, and S. Robinson, "Obesity in pregnancy," Journal of Obstetrics \& Gynaecology, vol. 113, no. 10, pp. 1117-1125, 2006.

[10] A. R. Amorim, S. Rossner, M. Neovius, P. M. Lourenco, and Y. Linne, "Does excess pregnancy weight gain constitute a major 
risk for increasing long-term BMI?" Obesity, vol. 15, no. 5, pp. 1278-1286, 2007.

[11] Y. Linne, L. Dye, B. Barkeling, and S. Rossner, "Long-term weight development in women: a 15-year follow-up of the effects of pregnancy," Obesity, vol. 12, no. 7, pp. 1166-1178, 2004.

[12] P. J. Salsberry and P. B. Reagan, "Taking the long view: the prenatal environment and early adolescent overweight," Research in Nursing \& Health, vol. 30, no. 3, pp. 297-307, 2007.

[13] C. Jevitt, I. Hernandez, and M. Groër, "Lactation complicated by overweight and obesity: supporting the mother and newborn," Journal of Midwifery \& Women's Health, vol. 52, no. 6, pp. 606-613, 2007.

[14] E. M. Perez-Pastor, B. S. Metcalf, J. Hosking, A. N. Jeffery, L. D. Voss, and T. J. Wilkin, "Assortative weight gain in motherdaughter and father-son pairs: an emerging source of childhood obesity. Longitudinal study of trios (EarlyBird 43)," International Journal of Obesity, vol. 33, no. 7, pp. 727-735, 2009.

[15] M. D. Levine, M. L. Klem, M. A. Kalarchian et al., "Weight gain prevention among women," Obesity, vol. 15, no. 5, pp. $1267-$ 1277, 2007.

[16] C. Lombard, A. Deeks, D. Jolley, and H. J. Teede, "Preventing weight gain: the baseline weight related behaviors and delivery of a randomized controlled intervention in community based women," BMC Public Health, vol. 9, no. 1, article 2, 2009.

[17] S. Kumanyika, R. W. Jeffery, A. Morabia, C. Ritenbaugh, and V. J. Antipatis, "Obesity prevention: the case for action," International Journal of Obesity, vol. 26, no. 3, pp. 425-436, 2002.

[18] C. Levitt, "Canadian perinatal surveillance system," Canadian Family Physician, vol. 44, pp. 583-584, 1998.

[19] R. M. Royall, "Model robust confidence intervals using maximum likelihood estimators," International Statistical Review, vol. 54, no. 2, pp. 221-226, 1986.

[20] StataCorp, Stata Statistical Software: Release 10. College Station, TX: Stata Corporation.

[21] M. Tjepkema, Adult Obesity in Canada: Measured Height and Weight, Statistics Canada, Ottawa, Canada, 2005.

[22] J. Gentleman, D. Blackwell, and M. Martin, "Obesity rate differences between U.S. and Canadian women and between U.S. and Canadian men: findings from the Joint Canada/ United States Survey of Health," Ottawa, Canada, Statistics Canada, 2006, http://www.statcan.gc.ca/bsolc/olc-cel/olc-cel? catno=11-522-X200600110395\&lang=eng.

[23] L. McLaren, "Socioeconomic status and obesity," Epidemiologic Reviews, vol. 29, no. 1, pp. 29-48, 2007.

[24] WHO, "Obesity: preventing and managing the global epidemic. Report of a WHO consultation," World Health Organization Technical Report Series, vol. 894, no. 1-12, pp. 1-253, 2000.

[25] A. H. Anis, W. Zhang, N. Bansback, D. P. Guh, Z. Amarsi, and C. L. Birmingham, "Obesity and overweight in Canada: an updated cost-of-illness study," Obesity Reviews, vol. 11, no. 1, pp. 31-40, 2010.

[26] P. M. Catalano and H. M. Ehrenberg, "The short- and longterm implications of maternal obesity on the mother and her offspring," Journal of Obstetrics \& Gynaecology, vol. 113, no. 10, pp. 1126-1133, 2006.

[27] M. Cedergren, "Effects of gestational weight gain and body mass index on obstetric outcome in Sweden," International Journal of Gynecology \& Obstetrics, vol. 93, no. 3, pp. 269-274, 2006.

[28] B. H. Wrotniak, J. Shults, S. Butts, and N. Stettler, "Gestational weight gain and risk of overweight in the offspring at age $7 \mathrm{y}$ in a multicenter, multiethnic cohort study," The American Journal of Clinical Nutrition, vol. 87, no. 6, pp. 1818-1824, 2008.

[29] M. Scharoun-Lee, J. S. Kaufman, B. M. Popkin, and P. GordonLarsen, "Obesity, race/ethnicity and life course socioeconomic status across the transition from adolescence to adulthood," Journal of Epidemiology \& Community Health, vol. 63, no. 2, pp. 133-139, 2009.

[30] A. Drewnowski and S. Specter, "Poverty and obesity: the role of energy density and energy costs," The American Journal of Clinical Nutrition, vol. 79, no. 1, pp. 6-16, 2004. 


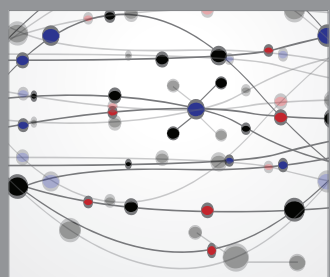

The Scientific World Journal
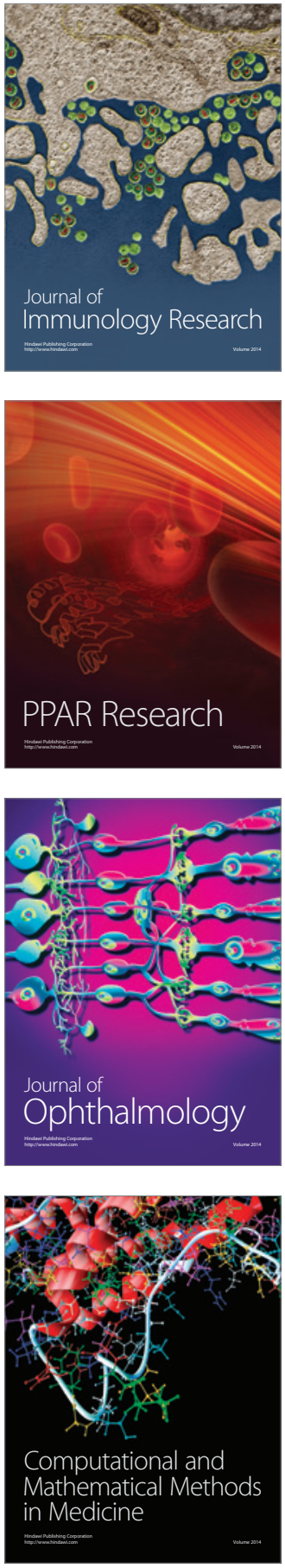

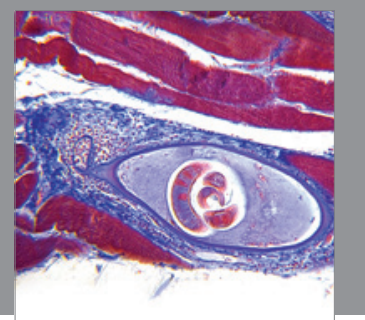

Gastroenterology

Research and Practice
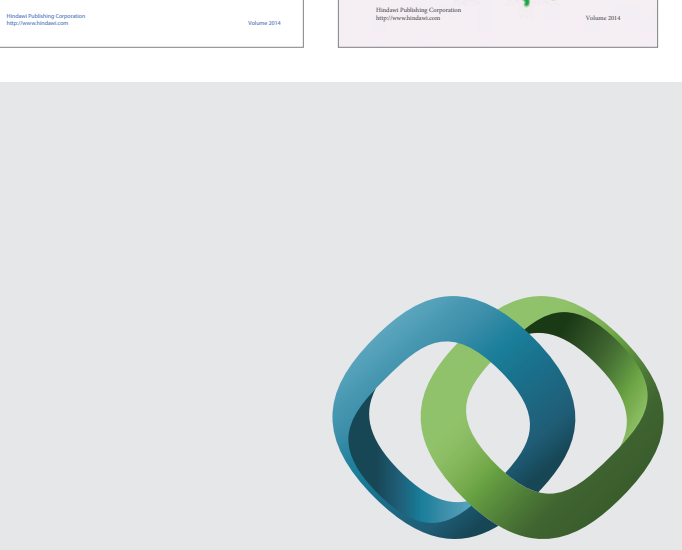

\section{Hindawi}

Submit your manuscripts at

http://www.hindawi.com
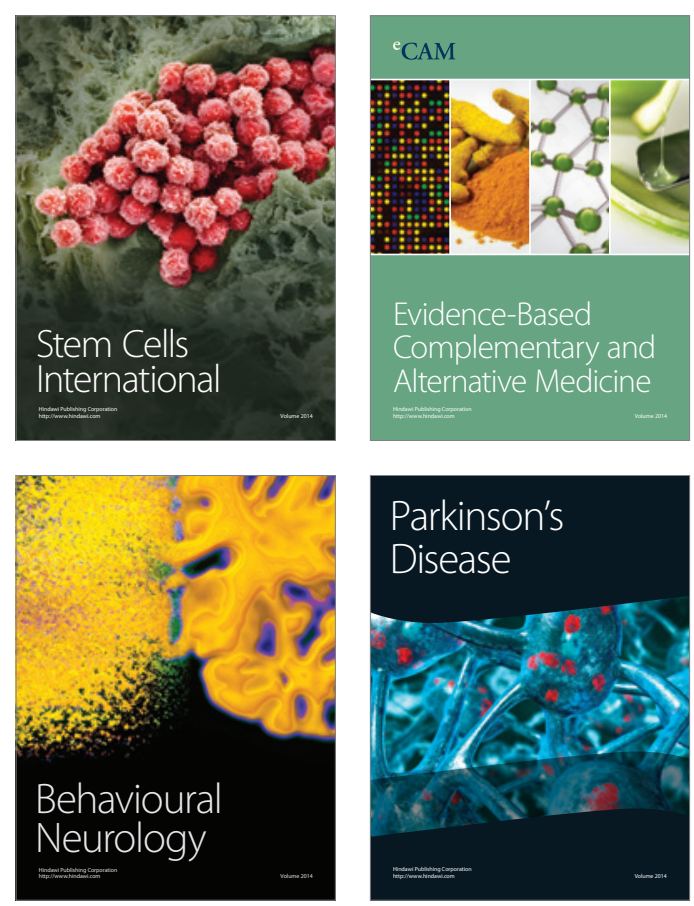

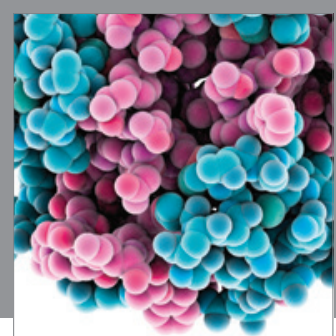

Journal of
Diabetes Research

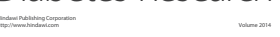

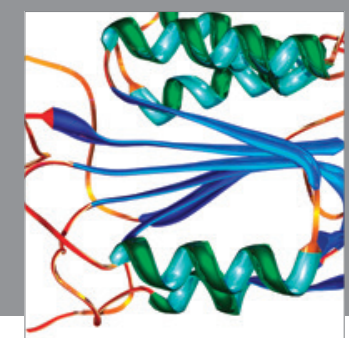

Disease Markers
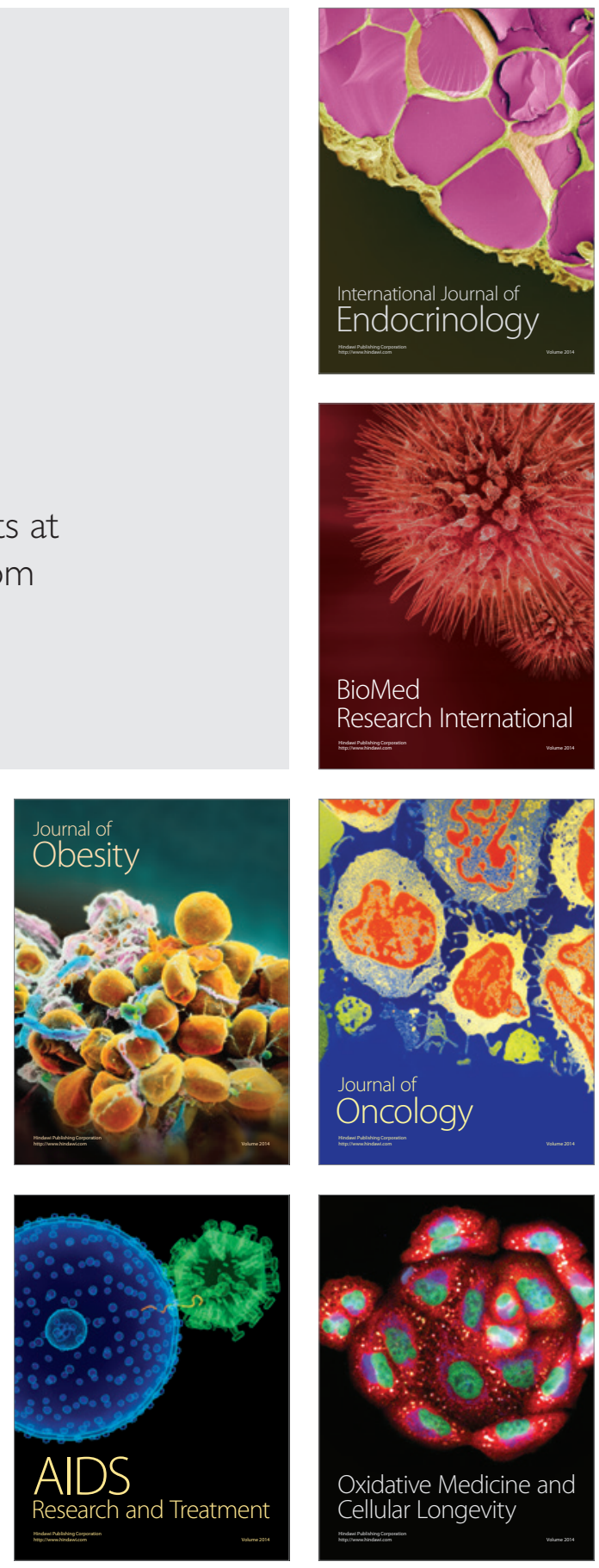